\title{
Torres de Hanói de 3 e 4 pinos: abordagens para o Ensino Básico
}

\section{Resumo}

Este trabalho mostra como explorar diversos conceitos matemáticos usando o jogo clássico Torres de Hanói com 3 pinos, e também como encontrar a solução para uma de suas variantes com 4 pinos. Para o caso clássico de 3 pinos, investigamos uma fórmula matemática que expressa a quantidade mínima de jogadas necessárias para vencer o jogo em função da quantidade de discos usando recorrências/recursividade, modelagem matemática e progressões geométricas. Apresentamos também fórmulas para o número de vezes que um disco ocupa cada um dos pinos durante a solução ideal do quebra-cabeça, ou seja, a que utiliza a menor quantidade de jogadas possíveis. Para a variante do jogo com 4 pinos, conhecida como quebra-cabeça de Reve, encontraremos uma fórmula fechada que expressa a quantidade mínima de jogadas para vencer o jogo em função do número de discos.

Palavras-chave: Torre de Hanói com 3 pinos; Torre de Hanói com 4 pinos; Recorrência; Quantidade Mínima de Movimentos; Modelagem Matemática.

\begin{abstract}
In this work we show how to explore mathematical concepts using the classical puzzle Tower of Hanói with 3 pegs, and how to find the solution for one of the variations with 4 pegs. For the classical case with 3 pegs, we investigated a mathematical formula that express the minimum amount of moves required to win the game based on the number of disks, we use recurrences, mathematical modeling and geometric progressions. We also present formulas for the number of times a disc occupies each one of the pegs during the ideal puzzle solution, that is, the one that uses the least amount of moves possible. For the variation with 4 pegs, known Reve's puzzle, we find a closed formulae that express the minimum quantity of moves to win the game.
\end{abstract}

Keywords: Tower of Hanói with 3 pegs; Tower of Hanói with 4 pegs; Recurrences; Minimum Amount of Moves; Mathematical Modeling.

\section{Introdução}

O quebra cabeça as Torres de Hanói ficou famoso por meio do matemático francês Édouard Lucas no ano de 1893, na obra Récréations Mathématiques, volume III, [6]. Lucas conheceu-o através do

\footnotetext{
${ }^{1}$ Parcialmente apoiada pela Capes
} 
amigo e professor N. Claus (do Sião), que fora apresentado ao quebra-cabeça em uma das suas viagens ao Vietnã, especificamente na região de Tonkin.

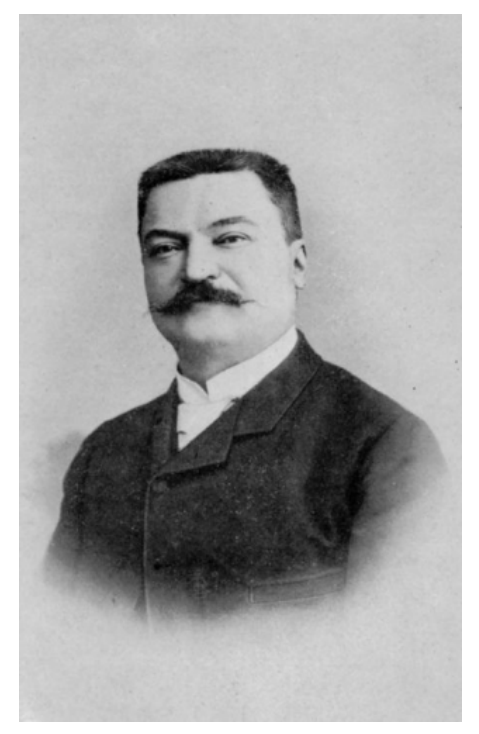

Figura 1: Édouard Lucas.

Lucas estimou o tempo necessário para resolver o jogo considerando um movimento por segundo. Com 64 discos, seriam necessários 18.446.744.073.709.551.615 movimentos, o que equivale a bilhões de séculos para resolver sem pausas. Além disso, Lucas comparou a solução matemática com um quebra-cabeça muito famoso da China, o Baguenaudier, que é um quebra-cabeça de desemaranhamento de anéis.

Logo após a descoberta do jogo, surgiram outras variações de desafios no início do século XX, sendo a mais famosa as Torres de Hanói com 4 pinos citada na obra The Canterbury Puzzle do especialista em quebra-cabeças e matemático Henry Ernest Dudeney, [2]. 87

Há muitas curiosidades envolvidas nesse jogo, por exemplo: a semelhança entre o Triângulo de Sierpinsk e um grafo representando os possíveis movimentos dos discos da Torre, ver em [9]. Para o caso clássico, em uma solução para o jogo utilizando a menor quantidade de movimentos possível, suponha que essa seja interrompida em uma jogada i,; em [3] ou [8] é descrita a configuração da torre nesse instante, isto é, em qual pino cada um dos discos estará. Mais curiosidades e detalhes sobre o assunto no livro [4].

O quebra-cabeça Torres de Hanói é composto por uma base e três pinos; chamaremos esses de A, B e C. No pino A há discos empilhados com diferentes tamanhos, em sequência do maior para o menor, como mostra a figura a seguir: 


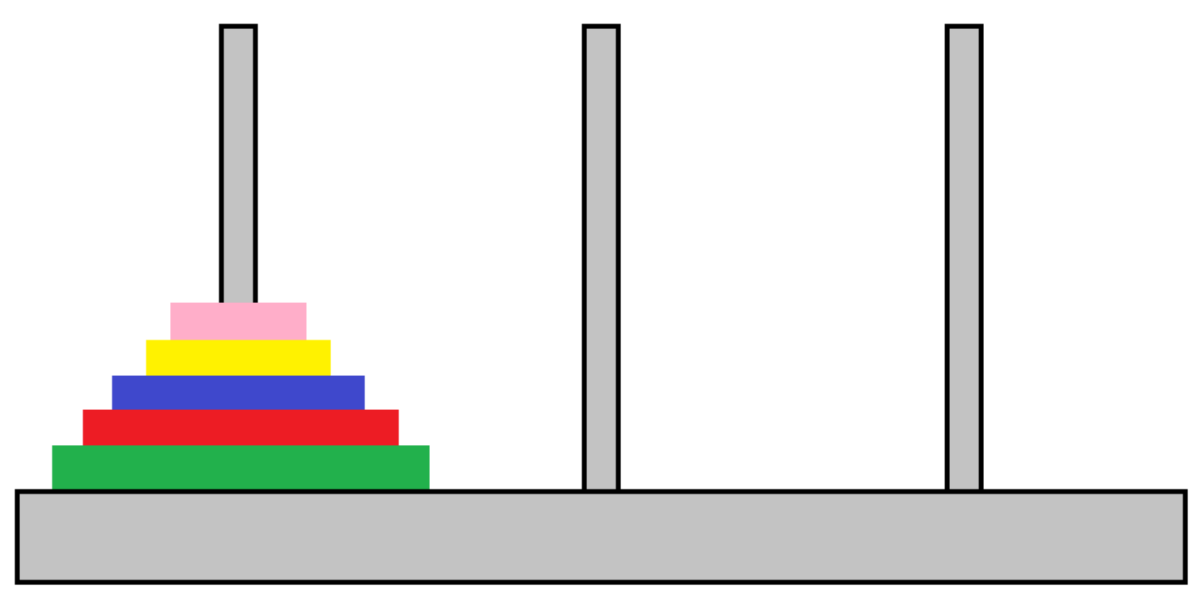

Figura 2: Torre de Hanói com 3 pinos.

O objetivo do jogo é transferir a pilha de discos do pino inicial A para o pino C, usando as regras a seguir:

- Apenas um disco pode ser movido por vez.

- Nenhum disco pode ficar em cima de um de raio menor.

- Apenas o disco do topo pode ser movido.

Nesse artigo, exploramos as ferramentas matemáticas no estudo da quantidade mínima de movimentos necessários para vencer o quebra-cabeça com n discos nos dois casos, com 3 e 4 pinos, e também quantas vezes cada disco se mover-se-á para os pinos A, B e C, caso clássico com 3 pinos. Consideramos as sequências numéricas $\left\{a_{n}\right\}$, onde $a_{n}$ é a quantidade mínima de movimentos necessários para transferir os $\mathrm{n}$ discos de um pino $\mathrm{A}$ para $\mathrm{C}$ quando possuirmos 3 pinos e $\left\{\mathrm{b}_{\mathrm{n}}\right\}$, para 4 pinos. Estamos à procura de uma expressão para $\mathrm{a}_{\mathrm{n}}$ e $\mathrm{b}_{\mathrm{n}}$.

\section{Torre com 3 pinos}

\subsection{Recursão}

Suponha que temos os $\mathrm{n}$ discos empilhados no pino A em forma de cone e desejamos transferi-los para o pino C. Observe que o último disco, ou seja, o maior de todos, será movido após retirarmos os $n-1$ discos que estão em cima dele, para isso usaremos $a_{n-1}$ movimentos e os colocaremos no pino B. Então, transferimos o maior disco para o pino $\mathrm{C}$ usando 1 movimento, e para finalizar realocamos os $\mathrm{n}-1$ discos no pino $\mathrm{B}$ para o pino $\mathrm{C}$, usando mais $\mathrm{a}_{\mathrm{n}-1}$ movimentos e concluímos o jogo. Para isso, efetuamos $a_{n-1}+1+a_{n-1}$ movimentos no total, ou seja,

$$
\mathrm{a}_{\mathrm{n}}=2 \mathrm{a}_{\mathrm{n}-1}+1 \text { e } \mathrm{a}_{1}=1 \text {, }
$$

pois com 1 disco temos apenas 1 movimento, que seria retirá-lo do pino A e colocá-lo do pino C. Acabamos de obter uma forma recursiva para a sequência $\left(a_{n}\right)$. 


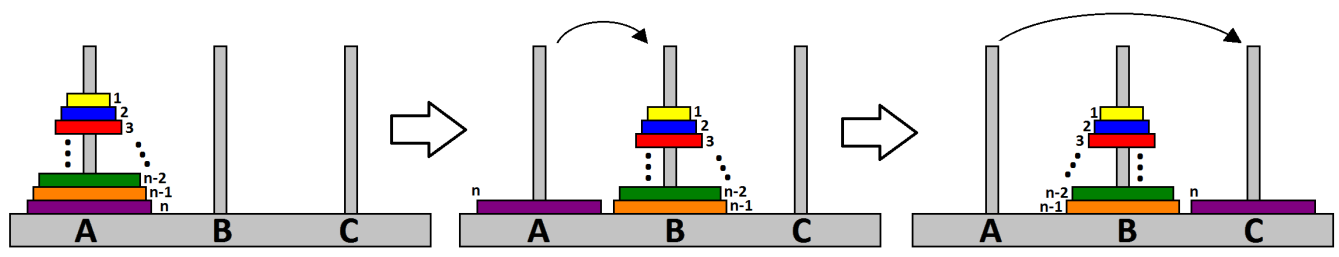

Figura 3: Solução com n discos.

Para uma torre com 1 disco é necessário 1 movimento apenas, daí a $a_{1}=1$ :
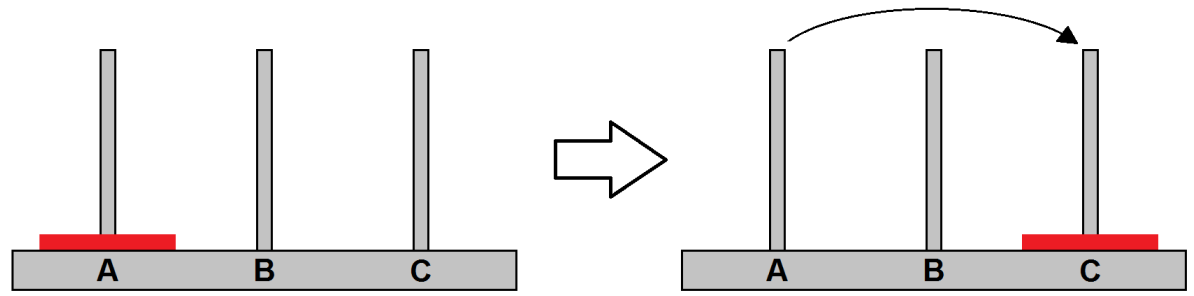

Figura 4: Solução com 1 disco.

Para uma torre com 2 discos são necessários 3 movimentos:

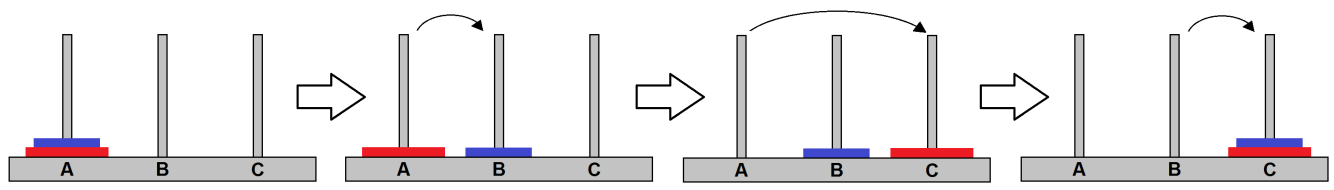

Figura 5: Solução com 3 discos.

Logo, $\mathrm{a}_{2}=2 \mathrm{a}_{1}+1=2.1+1=3$. Com 3 discos, substituímos $\mathrm{a}_{3}=2 \mathrm{a}_{2}+1=2.3+1=7$ : 

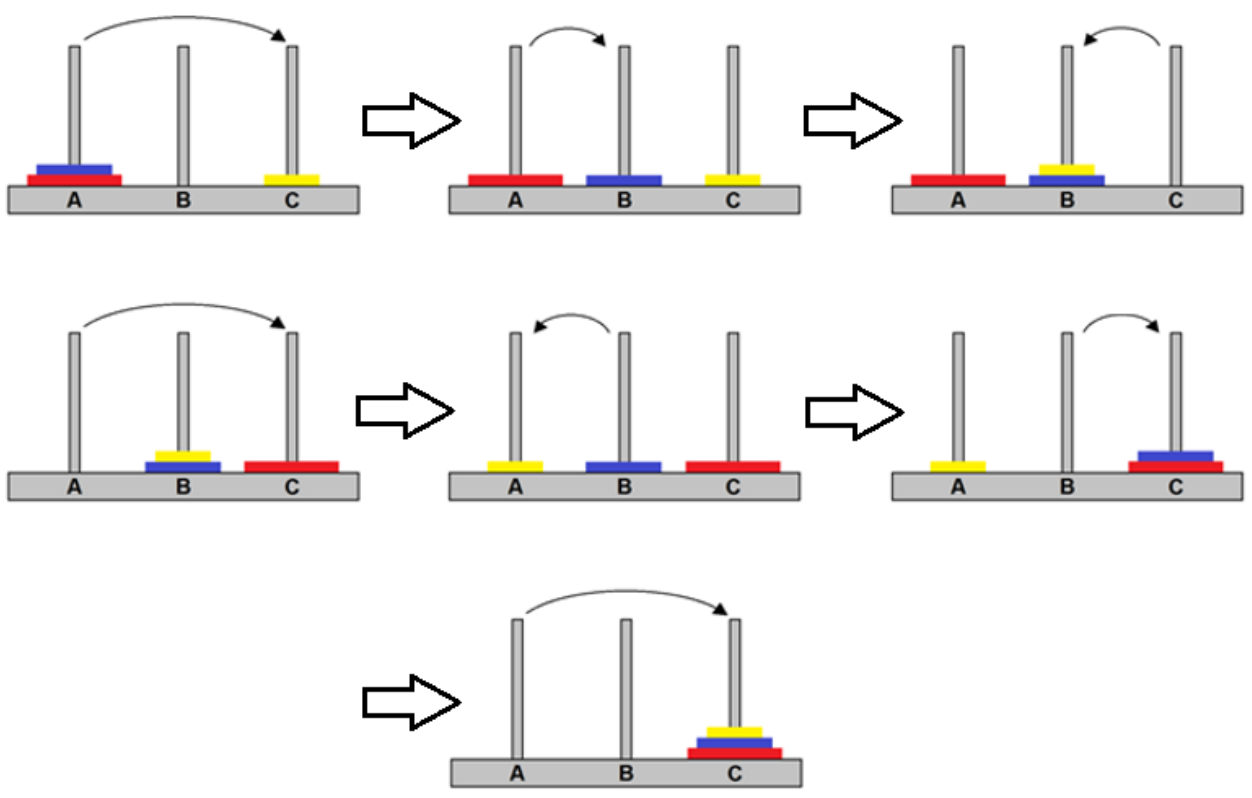

Figura 6: Solução com 3 discos.

Intuitivamente, percebemos que o maior disco move-se 1 vez, o segundo maior 2 vezes, o disco seguinte 4 vezes, ou seja, cada disco tem o dobro de movimentos do seu antecessor. Sendo assim, podemos escrever:

$$
a_{n}=2^{n-1}+2^{n-2}+\ldots+2+1
$$

que é a soma de uma Progressão Geométrica com n termos, primeiro termo $2^{\mathrm{n}-1}$ e razão $2^{-1}$.

Assim,

$$
a_{n}=\frac{2^{n-1}\left(1-2^{-n}\right)}{1-2^{-1}} \Rightarrow a_{n}=2^{n}-1
$$

para todo n natural, obtemos uma expressão fechada para $a_{n}$.

\subsection{Indução Matemática}

Note que essa argumentação foi apenas intuitiva. Uma prova formal para esse fato pode ser obtida usando Indução Matemática, que é uma ferramenta poderosíssima em demonstrações de proposições no conjunto dos números naturais. Em resumo, seja uma proposição ou propriedade que desejamos mostrar ser satisfeita para todos os números naturais. Tal proposição é verdadeira para $n=1$. Se, por hipótese, a proposição for satisfeita para um certo n, e isso implicar em ser satisfeita para $n+1$, então a proposição é válida para todo n natural.

Queremos provar que $a_{n}=2^{n}-1$ para todo $n$ natural. Para $n=1$, temos $a_{1}=1=2^{1}-1$; $\operatorname{logo}$, a proposição é verdadeira para $n=1$. Suponha, por hipótese de indução, que $a_{n}=2^{n}-1$ para algum 
natural n. Agora, suponha que temos $n+1$ discos, e queremos saber quanto vale $a_{n+1}$. Vimos na fórmula recursiva que $\mathrm{a}_{\mathrm{n}+1}=2 \mathrm{a}_{\mathrm{n}}+1$, e, então,

$$
\mathrm{a}_{\mathrm{n}+1}=2 \mathrm{a}_{\mathrm{n}}+1=2\left(2^{\mathrm{n}}-1\right)+1=2^{\mathrm{n}+1}-1 .
$$

Logo, se supusermos a proposição válida para $n$, então ela vale para $n+1$. Desse modo, por Indução Matemática, ela é válida para todo n natural.

\subsection{Modelagem Matemática}

Uma outra maneira de encontrar o termo geral de e ao mesmo tempo abordar o assunto funções é usar modelagem matemática, ou seja, estudar o comportamento da sequência e encontrar uma função elementar que a descreva. De modo geral, toda sequência numérica $\left(\mathrm{a}_{\mathrm{n}}\right)$ é uma função com domínio nos naturais e imagem nos reais. Procuramos $f: \mathbb{R} \rightarrow \mathbb{R}$ dentre as funções estudadas no ensino básico (polinomial, trigonométrica, logaritmica e exponencial), uma que coincida com a sequência, isto é, $a_{n}=f(n)$. Para facilitar, a partir de agora chamamos $a_{n}$ de $f(n)$. Considere a tabela abaixo, que representa as quantidades mínimas de movimentos necessários para vencer o jogo de acordo com a quantidade de discos:

\begin{tabular}{|c|c|}
\hline Número de discos & Quant. movimentos \\
\hline \hline 1 & 1 \\
\hline 2 & 3 \\
\hline 3 & 7 \\
\hline 4 & 15 \\
\hline 5 & 31 \\
\hline 6 & 63 \\
\hline 7 & 127 \\
\hline
\end{tabular}

Tabela 1: Número mínimo de jogadas em função do número de discos.

A partir dela, traçamos o gráfico de pontos cujo eixo das abscissas corresponde ao número de discos e o eixo das ordenadas à quantidade mínima de movimentos. 


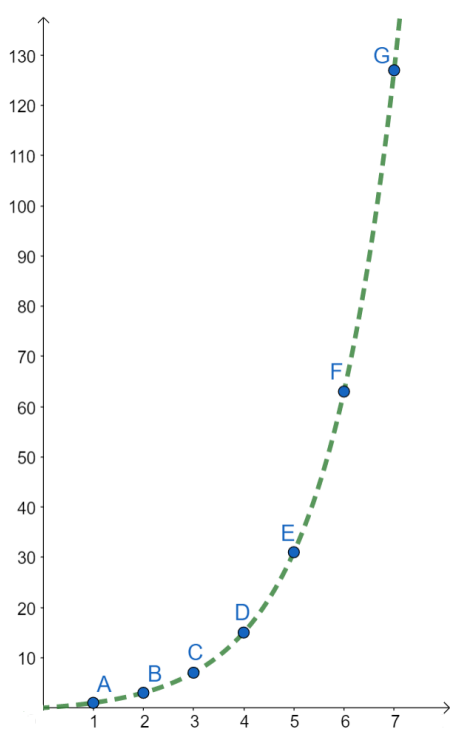

Figura 7: Gráfico ajustado.

Observando o gráfico, desconfiamos que seja uma polinomial ou função com crescimento exponencial. Primeiramente, observe, intuitivamente, que é crescente e injetiva pois quanto mais discos o jogo possui, maior a quantidade de movimentos necessária para vencer o jogo. Mostraremos agora que f não é do tipo polinomial.

Proposição 1. A função $\mathrm{f}(\mathrm{n})$, número mínimo de movimentos em função do número de discos $\mathrm{n}$, não é polinomial.

Demonstração. Suponha por contradição que $\mathrm{f}(\mathrm{n})=\sum_{\mathrm{p}=0}^{\mathrm{k}} \mathrm{b}_{\mathrm{p}} \mathrm{n}^{\mathrm{p}}$ é um polinômio de grau $\mathrm{k}$, com $\mathrm{b}_{\mathrm{k}} \neq 0$. Então:

$$
f(n+1)=\sum_{p=0}^{k} b_{p}(n+1)^{p}
$$


Subtraindo de (3) f(n), obtemos:

$$
\begin{aligned}
f(n+1)-f(n) & =\sum_{p=0}^{k} b_{p}(n+1)^{p}-\sum_{p=0}^{k} b_{p} n^{p} \\
& =\sum_{p=0}^{k} b_{p}\left((n+1)^{p}-n^{p}\right) \\
& =b_{k}\left((n+1)^{k}-n^{k}\right)+\sum_{p=0}^{k-1} b_{p}\left((n+1)^{p}-n^{p}\right) \\
& =b_{k}\left(n^{k}+\sum_{p=0}^{k-1}\left(\begin{array}{c}
k-1 \\
p
\end{array}\right) n^{k-1-p}-n^{k}\right)+\sum_{p=0}^{k-1} b_{p}\left((n+1)^{p}-n^{p}\right) \\
& =b_{k}\left(\sum_{p=0}^{k-1}\left(\begin{array}{c}
k-1 \\
p
\end{array}\right) n^{k-1-p}\right)+\sum_{p=0}^{k-1} b_{p}\left((n+1)^{p}-n^{p}\right),
\end{aligned}
$$

que é um polinômio de grau $k-1$. Por recorrência, vimos que $f(n)=2 \cdot f(n-1)+1$; logo, $f(n+1)=2 \cdot f(n)+1$. Assim, $f(n+1)-f(n)=f(n)+1$. Se f fosse uma polinomial de grau $k$, então o grau de $f(n+1)-f(n)$ teria o mesmo grau de $f(n)+1$, que é k. O que não ocorre em polinômios. Logo, f não é uma função polinomial.

Uma outra demonstração da Proposição 1 é por meio de derivadas de ordem superior. Vejamos a seguir.

Demonstração. Seja $\mathrm{f}(\mathrm{n})=\sum_{\mathrm{p}=0}^{\mathrm{k}} \mathrm{b}_{\mathrm{p}} \mathrm{n}^{\mathrm{p}}$ um polinômio de grau $\mathrm{k}$, com $\mathrm{b}_{\mathrm{k}} \neq 0$.

Sabendo que $f(n)=2 \cdot f(n-1)+1$ e derivando $k$ vezes $f$, obtemos:

$$
\mathrm{f}^{(\mathrm{k})}(\mathrm{n})=\mathrm{k} ! \cdot \mathrm{b}_{\mathrm{k}} \text {. }
$$

Assim, temos:

$$
\begin{aligned}
\mathrm{f}^{(\mathrm{k})}(\mathrm{n}) & =2 \cdot \mathrm{f}^{(\mathrm{k})}(\mathrm{n}-1) \\
\Rightarrow \mathrm{k} ! \cdot \mathrm{b}_{\mathrm{k}} & =2 \cdot \mathrm{k} ! \cdot \mathrm{b}_{\mathrm{k}} \\
\Rightarrow \mathrm{b}_{\mathrm{k}} & =2 \cdot \mathrm{b}_{\mathrm{k}} .
\end{aligned}
$$

Essa igualdade só é verdadeira se $b_{k}=0$, o que contraria a nossa hipótese. Portanto, função f não pode ser um polinômio.

Proposição 2. A função $\mathrm{f}(\mathrm{n})$, número mínimo de movimentos em função do número $\mathrm{n}$ discos, não é logarítmica.

Demonstração. Seja $\mathrm{f}(\mathrm{n})=\log _{\mathrm{a}}(\mathrm{bn}+\mathrm{c})+\mathrm{d}, 8 \mathrm{a}, \mathrm{b}, \mathrm{c}, \mathrm{d} \in \mathbb{R}$, onde $\mathrm{a} \neq 1$ e a $>0$. 
Utilizando a relação da recorrência (1), temos:

$$
\begin{aligned}
\mathrm{f}(\mathrm{n}+1) & =2 \cdot \mathrm{f}(\mathrm{n})+1 \\
\Rightarrow \log _{\mathrm{a}}(\mathrm{b}(\mathrm{n}+1)+\mathrm{c})+\mathrm{d} & =2 \cdot\left(\log _{\mathrm{a}}(\mathrm{bn}+\mathrm{c})+\mathrm{d}\right)+1 \\
\Rightarrow \log _{\mathrm{a}}(\mathrm{b}(\mathrm{n}+1)+\mathrm{c})+\mathrm{d} & =2 \cdot \log _{\mathrm{a}}(\mathrm{bn}+\mathrm{c})+2 \mathrm{~d}+1 \\
\Rightarrow \log _{\mathrm{a}}(\mathrm{b}(\mathrm{n}+1)+\mathrm{c}) & =\log _{\mathrm{a}}(\mathrm{bn}+\mathrm{c})^{2}+\mathrm{d}+1 \\
\Rightarrow \log _{\mathrm{a}}(\mathrm{b}(\mathrm{n}+1)+\mathrm{c})-\log _{\mathrm{a}}(\mathrm{bn}+\mathrm{c})^{2} & =\mathrm{d}+1 \\
\Rightarrow \log _{\mathrm{a}}\left(\frac{(\mathrm{b}(\mathrm{n}+1)+\mathrm{c}}{(\mathrm{bn}+\mathrm{c})^{2}}\right) & =\mathrm{d}+1 \\
\Rightarrow \mathrm{a}^{\mathrm{d}+1} & =\frac{\mathrm{b}(\mathrm{n}+1)+\mathrm{c}}{(\mathrm{bn}+\mathrm{c})^{2}} \\
\Rightarrow \mathrm{a}^{\mathrm{d}+1} \cdot(\mathrm{bn}+\mathrm{c})^{2} & =\mathrm{b}(\mathrm{n}+1)+\mathrm{c} \\
\Rightarrow \mathrm{a}^{\mathrm{d}+1} \mathrm{~b}^{2} \mathrm{n}^{2}+2 \mathrm{a}^{\mathrm{d}+1} \mathrm{bcn}+\mathrm{c}^{2} \mathrm{a}^{\mathrm{d}+1} & =\mathrm{bn}+\mathrm{b}+\mathrm{c} .
\end{aligned}
$$

Igualando os coeficientes de mesma potência temos:

$$
\begin{aligned}
\mathrm{a}^{\mathrm{d}+1} \mathrm{~b}^{2} & =0, \\
2 \mathrm{a}^{\mathrm{d}+1} \mathrm{bc} & =\mathrm{b} \quad \mathrm{e} \\
\mathrm{c}^{2} \mathrm{a}^{\mathrm{d}+1} & =\mathrm{b}+\mathrm{c} .
\end{aligned}
$$

De (4), temos que $\mathrm{a}=0$ ou $\mathrm{b}=0$. Não é possível $\mathrm{a}=0$, pois contraria a definição de logaritmo; $\log \mathrm{o}, \mathrm{b}=0$. Assim, $\mathrm{f}(\mathrm{n})=\log _{\mathrm{a}}(\mathrm{c})+\mathrm{d}$ é constante, mas f não é constante, logo, não pode ser desse tipo.

Continuando a nossa análise, vamos verificar se f é exponencial. Analisando o comportamento das derivadas de ordem superior da função polinomial $f^{(k)}(n)=2 \cdot f^{(k)}(n-1)$, vemos que a função relacionada ao jogo é uma função de crescimento exponencial. Logo, a função $\mathrm{f}(\mathrm{n})=\mathrm{k} \cdot \mathrm{a}^{\mathrm{n}}+\mathrm{b}$, tal que $\mathrm{k}, \mathrm{b}$ e $\mathrm{a} \in \mathbb{R}$ constantes, com $\mathrm{a} \neq 1$, é a função candidata que procuramos.

Proposição 3. A função $\mathrm{f}(\mathrm{n})$, número mínimo de movimentos em função do número $\mathrm{n}$ de discos não é exponencial, isto é, não tem a forma $\mathrm{f}(\mathrm{x})=\mathrm{b}^{\mathrm{x}}, 0<\mathrm{b} \neq 1$, e sim de crescimento exponencial.

Demonstração. De acordo com [5], as funções exponenciais têm, exclusivamente, as seguintes propriedades:

1. $f(x) \cdot f(y)=f(x+y)$

2. $f(x)=b^{x}, 8 x \in \mathbb{R}$, onde $f(1)=b$

3. $f(n x)=f(x)^{n}$

4. $x<y \Rightarrow f(x)<f(y), b>1$

$\mathrm{x}<\mathrm{y} \Rightarrow \mathrm{f}(\mathrm{y})<\mathrm{f}(\mathrm{x}), 0<\mathrm{b}<1$

Sabemos que para vencer o jogo com 1 disco é necessário apenas 1 movimento, ou seja, $\mathrm{f}(1)=1$. Com 2 discos, são necessários 3 movimentos então, $\mathrm{f}(2)=3$. Com 3 discos, são necessários 7 movimentos, ou seja, $\mathrm{f}(3)=7$.

Observando as características da definição de função exponencial, vemos que obedece apenas uma das propriedades: 
1. $\mathrm{f}(1) \cdot \mathrm{f}(2)=1 \cdot 3=\mathrm{f}(1+2)=7$ (falso)

2. $\mathrm{f}(\mathrm{x})=\mathrm{b}^{\mathrm{x}}, 8 \mathrm{x} \in \mathbb{N}$, onde $\mathrm{f}(1)=\mathrm{b}=1$ (falso)

3. $\mathrm{f}(2 \cdot 1)=\mathrm{f}(1)^{2}$ (falso)

4. $1<2 \Rightarrow \mathrm{f}(1)<\mathrm{f}(2), \mathrm{b}>1$ (verdadeiro)

O que mostra claramente que a função não é do tipo $f(n)=b^{n}$. Resta-nos analisar a função da família da exponencial mais geral, a do tipo $\mathrm{f}(\mathrm{n})=\mathrm{k} \cdot \mathrm{b}^{\mathrm{n}}+\mathrm{c}, \mathrm{a}>0, \mathrm{~b} \neq 1, \mathrm{k} \neq 0, \mathrm{~b}, \mathrm{c}, \mathrm{k} \in \mathbb{R}$.

Pela relação da recorrência (1) sabemos que $f(n)=2 \cdot f(n-1)+1$, daí obtemos:

$$
\begin{aligned}
\mathrm{f}(\mathrm{n}) & =2 \cdot \mathrm{f}(\mathrm{n}-1)+1 \\
\Rightarrow \mathrm{k} \cdot \mathrm{a}^{\mathrm{n}}+\mathrm{b} & =2 \cdot\left(\mathrm{k} \cdot \mathrm{a}^{\mathrm{n}-1}+\mathrm{b}\right)+1 \\
\Rightarrow \mathrm{k} \cdot \mathrm{a}^{\mathrm{n}}+\mathrm{b} & =2 \cdot \mathrm{k} \cdot \mathrm{a}^{\mathrm{n}-1}+2 \cdot \mathrm{b}+1 \\
\Rightarrow \mathrm{k} \cdot \mathrm{a}^{\mathrm{n}} & =2 \cdot \mathrm{k} \cdot \mathrm{a}^{\mathrm{n}-1}+\mathrm{b}+1 \\
\Rightarrow \mathrm{k} \cdot \mathrm{a} \cdot \mathrm{a}^{\mathrm{n}-1} & =2 \cdot \mathrm{k} \cdot \mathrm{a}^{\mathrm{n}-1}+\mathrm{b}+1 .
\end{aligned}
$$

Da equação (7), vamos igualar os coeficiente dos dois lados. Temos que $2 \cdot \mathrm{k}=\mathrm{k} \cdot \mathrm{a} \Rightarrow \mathrm{a}=2 \mathrm{e}$ $\mathrm{b}+1=0 \Rightarrow \mathrm{b}=-1$. Portanto, a função $\mathrm{f}(\mathrm{n})=\mathrm{k} \cdot 2^{\mathrm{n}}-1$ é a procurada. Resta saber o valor de $\mathrm{k}$. Como $f(1)=1$, então $f(1)=k \cdot 2^{1}-1=1$. Logo, $2 \mathrm{k}=2$, implicando $\mathrm{k}=1$. Assim, a função que procuramos é

$$
f(n)=2^{n}-1 .
$$

\subsection{Progressão Geométrica}

Para finalizar essa seção, encontraremos uma expressão para f(n) usando progressões geométricas e a quantidade total de movimento de cada disco na solução do jogo.

Suponha uma torre com $\mathrm{n}$ discos. Se observarmos o movimento de cada disco no jogo, vemos que o maior disco move-se uma única vez. O disco $n-1$ move-se duas vezes: uma vez para ir ao pino $\mathrm{B}$, logo a seguir $\mathrm{n}-2$ discos serão colocados em cima dele, o maior disco fará seu único movimento para o pino $\mathrm{C}$, a seguir os $\mathrm{n}-1$ discos retornam ao pino $\mathrm{A}$, e o disco $\mathrm{n}-1$ fará seu segundo movimento para o pino $\mathrm{C}$, totalizando dois movimentos. O disco $\mathrm{n}-2$ fará o dobro de movimentos do disco $n-1$, totalizando 4 movimentos. E assim sucessivamente. Considere a tabela a seguir onde a primeira coluna é a quantidade total de discos e a segunda descreve a soma dos movimentos de cada disco, do maior (sempre 1) até o menor. 


\begin{tabular}{|c|c|}
\hline No discos & Quant. movimentos \\
\hline \hline 1 & 1 \\
\hline 2 & $1+2$ \\
\hline 3 & $1+2+4$ \\
\hline 4 & $1+2+4+8$ \\
\hline 5 & $1+2+4+8+16$ \\
\hline
\end{tabular}

Tabela 2: Número mínimo de jogadas para 5 discos em função da soma dos movimentos de cada disco

A tabela anterior mostra que a quantidade de movimentos de cada disco é o dobro do anterior, em especial potências de base 2, sendo que o maior disco tem apenas 1 movimento.

\begin{tabular}{|c|c|}
\hline $\mathbf{N}^{\mathbf{o}}$ discos & Quant. movimentos \\
\hline \hline 1 & $2^{0}$ \\
\hline 2 & $2^{1}+2^{0}$ \\
\hline 3 & $2^{2}+2^{1}+2^{0}$ \\
\hline 4 & $2^{3}+2^{2}+2^{1}+2^{0}$ \\
\hline 5 & $2^{4}+2^{3}+2^{2}+2^{1}+2^{0}$ \\
\hline
\end{tabular}

Tabela 3: Número mínimo de jogadas para 5 discos em função da soma dos movimentos de cada disco

Pra reforçar esse padrão, vamos utilizar novamente a Proposição , mostrando a quantidade mínima de movimentos para 6 discos:

$$
\begin{aligned}
a_{n} & =2 \cdot a_{n-1}+1 \\
\underbrace{2^{5}+2^{4}+2^{3}+2^{2}+2^{1}+2^{0}}_{6 \text { discos }} & =2 \cdot(\underbrace{\left(2^{0}+2^{1}+2^{2}+2^{3}+2^{4}\right.}_{5 \text { discos }})+1
\end{aligned}
$$

As tabelas anteriores mostram essa propriedade de uma maneira empírica. Vamos ver uma prova formal para um disco k. Intuímos que, para uma Torre de Hanói com n discos, a quantidade mínima de movimentos de um disco $\mathrm{k}$ será $2^{\mathrm{n}-\mathrm{k}}$.

Teorema 1. A quantidade de movimentos de um disco $\mathrm{k}$ em função de $\mathrm{n}$ discos no total é dada por $\mathrm{d}_{\mathrm{n}, \mathrm{k}}=2^{\mathrm{n}-\mathrm{k}}, 1 \leq \mathrm{k} \leq \mathrm{n}$.

Demonstração. Seja $\mathrm{k}$ fixado tal que $\mathrm{k} \leq \mathrm{n}$. Para $\mathrm{n}=1$, então $\mathrm{k}=1$ e $\mathrm{d}_{1,1}=2^{1-1}=2^{0}=1$, que é verdadeiro, pois com 1 disco só ocorre 1 movimento. Suponha por hipótese que $\mathrm{d}_{\mathrm{n}, \mathrm{k}}=2^{\mathrm{n}-\mathrm{k}}$ para algum $\mathrm{k} \leq \mathrm{n}$. Provaremos que $\mathrm{d}_{\mathrm{n}+1, \mathrm{k}}=2^{\mathrm{n}+1-\mathrm{k}}$, para todo $\mathrm{k} \leq \mathrm{n}+1$. Se $\mathrm{k} \leq \mathrm{n}$, então, para movermos a torre com $\mathrm{n}+1$ discos do pino A para o pino $\mathrm{C}$, primeiro movemos a torre com $\mathrm{n}$ discos de $\mathrm{A}$ para $\mathrm{B}$; realizando $\mathrm{d}_{\mathrm{n}, \mathrm{k}}$ movimentos com o disco $\mathrm{k}$, por hipótese movemos o disco $\mathrm{k}+1$ para o pino $\mathrm{C}$, e depois movemos a torre com $\mathrm{n}$ discos de $\mathrm{B}$ para $\mathrm{C}$, realizando mais $2^{\mathrm{n}-\mathrm{k}}$ movimentos com o disco $\mathrm{k}$. Totalizamos $2 \cdot 2^{\mathrm{n}-\mathrm{k}}=2^{\mathrm{n}+1-\mathrm{k}}$.

Assim, $\mathrm{d}_{\mathrm{n}+1, \mathrm{k}}=2^{\mathrm{n}+1-\mathrm{k}}$ se $\mathrm{k} \leq \mathrm{n}$. Se $\mathrm{k}=\mathrm{n}+1$, então, $\mathrm{d}_{\mathrm{n}+1, \mathrm{n}+1}=2^{\mathrm{n}+1-(\mathrm{n}+1)}=2^{0}=1$ que é a quantidade de movimentos do disco $n+1$. 


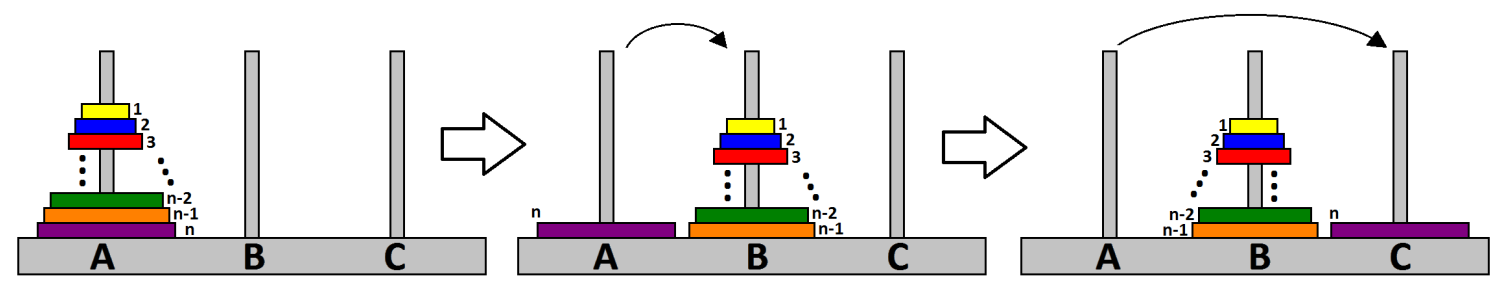

Figura 8: Solução da Torre com 3 pinos.

Agora que já sabemos que a quantidade de jogadas de cada disco é uma progressão geométrica de razão 2 , com o primeiro elemento valendo 1 , podemos encontrar a fórmula fechada da quantidade mínima de movimentos em função de n.

Aplicando a fórmula da soma $\mathrm{S}_{\mathrm{n}}$ de $\mathrm{n}$ termos de uma progressão geométrica, de $\mathrm{d}_{\mathrm{n}, 1}=1$ e razão igual a 2, temos:

$$
\mathrm{S}_{\mathrm{n}}=\frac{\mathrm{d}_{\mathrm{n}, 1} \cdot\left(2^{\mathrm{n}}-1\right)}{2-1}=\frac{1 \cdot\left(2^{\mathrm{n}}-1\right)}{2-1}=2^{\mathrm{n}}-1
$$

Logo, $a_{n}=S_{n}=2^{n}-1$.

\subsection{Número de vezes que um disco $k$ vai para os pinos $A, B$ ou $C$}

Nesta seção, iremos estudar, através de sequências e recorrências, o número de vezes que cada disco é movido para o pino inicial (A), o pino intermediário (B) e o pino final (C).

Sejam $A_{n, k}, B_{n, k}$ e $C_{n, k}$ a quantidade mínima de jogadas que um disco k vai para os pinos $A, B$ e $\mathrm{C}$, e $\mathrm{d}_{\mathrm{n}, \mathrm{k}}$ a quantidade de movimentos que do disco $\mathrm{k}$ para um total de $\mathrm{n}$ discos.

Primeiramente, vamos observar o padrão com 1, 2, 3, 4 e 5 discos no total.

\begin{tabular}{|c|c|c|c|c|}
\hline Disco k & A & B & C & Total \\
\hline Disco 1 & 0 & 0 & 1 & 1 \\
\hline
\end{tabular}

Tabela 4: Número de vezes que um disco vai para os pinos

Como temos apenas 1 disco, então esse só é movimentado uma vez para o pino $\mathrm{C}$, ou seja, $\mathrm{A}_{1,1}=0$, $\mathrm{B}_{1,1}=0$ e $\mathrm{C}_{1,1}=1$. Para 2 discos temos a Tabela 5 .

\begin{tabular}{|c|c|c|c|c|}
\hline Disco k & A & B & C & Total \\
\hline Disco 2 & 0 & 0 & 1 & 1 \\
\hline Disco 1 & 0 & 1 & 1 & 2 \\
\hline
\end{tabular}

Tabela 5: Número de vezes que os discos 1 e 2 ocupam os pinos

O maior sempre vai primeiramente para o pino final $\mathrm{C}$, enquanto o disco menor vai para o pino $\mathrm{B}$ e C, uma vez para cada. Usando a notação, teremos: 


$$
\begin{aligned}
& A_{2,2}=0, B_{2,2}=0 \text { e } C_{2,2}=1 . \\
& A_{2,1}=0, B_{2,1}=1 \text { e } C_{2,2}=1 .
\end{aligned}
$$

Vejamos agora uma tabela para 3 discos no total:

\begin{tabular}{|c|c|c|c|c|}
\hline Disco k & A & B & C & Total \\
\hline Disco 3 & 0 & 0 & 1 & 1 \\
\hline Disco 2 & 0 & 1 & 1 & 2 \\
\hline Disco 1 & 1 & 1 & 2 & 4 \\
\hline
\end{tabular}

Tabela 6: Número de vezes que os discos 1, 2 e 3 ocupam os pinos

$$
\begin{aligned}
& \mathrm{A}_{3,3}=0, \mathrm{~B}_{3,3}=0 \text { e } \mathrm{C}_{3,3}=1 . \\
& \mathrm{A}_{3,2}=0, \mathrm{~B}_{3,2}=1 \text { e } \mathrm{C}_{3,2}=1 . \\
& \mathrm{A}_{3,1}=1, \mathrm{~B}_{3,1}=1 \text { e } \mathrm{C}_{3,1}=2 .
\end{aligned}
$$

Observe que a soma das quantidades das jogadas do disco $\mathrm{k}$ em cada pino resulta na quantidade total de movimentos do disco $\mathrm{k}$, que pelo Teorema 1 é $2^{\mathrm{n}-\mathrm{k}}$, ou seja:

$$
\mathrm{A}_{\mathrm{n}, \mathrm{k}}+\mathrm{B}_{\mathrm{n}, \mathrm{k}}+\mathrm{C}_{\mathrm{n}, \mathrm{k}}=2^{\mathrm{n}-\mathrm{k}}
$$

Teorema 2. Considere o jogo com $\mathrm{n}$ discos. A quantidade de vezes que o disco $k$ ocupa os pinos A, B e C, respectivamente, durante a solução do jogo é dada por:

$$
\begin{aligned}
\mathrm{C}_{\mathrm{n}, \mathrm{k}} & =\frac{2^{\mathrm{n}+1-\mathrm{k}}+3+(-1)^{\mathrm{n}+\mathrm{k}}}{6}, \\
\mathrm{~B}_{\mathrm{n}, \mathrm{k}} & =\frac{2^{\mathrm{n}-\mathrm{k}}+(-1)^{\mathrm{n}+\mathrm{k}+1}}{3} \mathrm{e} \\
\mathrm{A}_{\mathrm{n}, \mathrm{k}} & =\frac{2^{\mathrm{n}+1-\mathrm{k}}-3+(-1)^{\mathrm{n}+\mathrm{k}}}{6} .
\end{aligned}
$$

Demonstração. Usaremos Indução Matemática, primeiro mostraremos que as três fórmulas anteriores são verdadeiras para $n=1$; por hipótese de indução suporemos que são verdadeiras para um $\mathrm{n}$ qualquer, e mostraremos que valem para $\mathrm{n}+1$ discos, isto é,

$$
\begin{aligned}
& A_{n+1, k}=\frac{2^{n+2-k}-3+(-1)^{n+k+1}}{6} \\
& B_{n+1, k}=\frac{2^{n+1-k}+(-1)^{n+k+2}}{3} e \\
& C_{n+1, k}=\frac{2^{n+2-k}+3+(-1)^{n+k+1}}{6} .
\end{aligned}
$$

Para $\mathrm{n}=1$, temos que $\mathrm{k}=1$, e

$$
\begin{aligned}
& A_{1,1}=\frac{2^{1+1-1}-3+(-1)^{1+1}}{6}=0 \\
& B_{1,1}=\frac{2^{1-1}+(-1)^{1+1+1}}{3}=0 \\
& C_{1,1}=\frac{2^{1+1-1}+3+(-1)^{1+1}}{6}=1 .
\end{aligned}
$$


Logo, as três fórmulas valem para $n=1$.

Suponha que sejam válidas para $n$ discos, e vamos provar que valem para $n+1$ discos. Para mover $\mathrm{n}+1$ discos para o pino $\mathrm{C}$, primeiro movemos os $\mathrm{n}$ discos menores para o pino $\mathrm{B}$, o maior disco para o pino $\mathrm{C}$, e por último os n discos que estão em B para $\mathrm{C}$ também.

Observe que $A_{n, k}$ é a quantidade de vezes que o disco $\mathrm{k}$ passa por $\mathrm{A}$ ao movermos a pilha de $\mathrm{n}$ discos de A para C. Assim, $A_{n, k}$ é também a quantidade de vezes que o disco $\mathrm{k}$ passa por $\mathrm{B}$ ao movermos a pilha de $\mathrm{n}$ discos de $\mathrm{B}$ para $\mathrm{C}$. Como $\mathrm{B}_{\mathrm{n}, \mathrm{k}}$ é a quantidade de vezes que o disco $\mathrm{k}$ passa por $\mathrm{B}$ ao mover a pilha de $\mathrm{n}$ discos de $\mathrm{A}$ para $\mathrm{C}, \mathrm{B}_{\mathrm{n}, \mathrm{k}}$ é também a quantidade de vezes que o disco k passa por $\mathrm{C}$ ao mover a pilha de $\mathrm{n}$ discos de $\mathrm{A}$ para B. Seguindo o raciocínio, $\mathrm{C}_{\mathrm{n}, \mathrm{k}}$ é a quantidade de vezes que o disco $\mathrm{k}$ movimenta-se para o pino $\mathrm{C}$ ao transferirmos os $\mathrm{n}$ discos de $\mathrm{A}$ para $\mathrm{C}$, e é também a quantidade de vezes que o mesmo disco k passa por B ao movermos os $\mathrm{n}$ discos de A para B.

Para sabermos quantas vezes um disco k se movimentar-se-á para o pino A durante esse processo de transferir $n+1$ discos de $\mathrm{A}$ para $\mathrm{C}$, calculamos quantas vezes ele se move para $\mathrm{A}$ ao colocarmos os $n$ discos em $B$, e somamos às quantidades de vezes que se move em $A$ para transferir os $n$ discos de $B$ para $C: A_{n+1, k}=A_{n, k}+B_{n, k}$. Da mesma forma, $B_{n+1, k}=A_{n, k}+C_{n, k}$ e $C_{n+1, k}=B_{n, k}+C_{n, k}$. Assim, se as fórmulas valem para $n$, podemos mostrar que valem para $n+1$.

$$
\begin{aligned}
A_{\mathrm{n}+1, \mathrm{k}} & =\mathrm{A}_{\mathrm{n}, \mathrm{k}}+\mathrm{B}_{\mathrm{n}, \mathrm{k}} \\
& =\frac{2^{\mathrm{n}+1-\mathrm{k}}-3+(-1)^{\mathrm{n}+\mathrm{k}}}{6}+\frac{2^{\mathrm{n}-\mathrm{k}}+(-1)^{\mathrm{n}+\mathrm{k}+1}}{3} \\
& =\frac{2^{\mathrm{n}+1-\mathrm{k}}-3+(-1)^{\mathrm{n}+\mathrm{k}}}{6}+\frac{2 \cdot 2^{\mathrm{n}-\mathrm{k}}+2 \cdot(-1)^{\mathrm{n}+\mathrm{k}+1}}{6} \\
& =\frac{2^{\mathrm{n}+1-\mathrm{k}}-3+(-1)^{\mathrm{n}+\mathrm{k}}}{6}+\frac{2^{\mathrm{n}+1-\mathrm{k}}+2 \cdot(-1)^{\mathrm{n}+\mathrm{k}+1}}{6} \\
& =\frac{2 \cdot 2^{\mathrm{n}+1-\mathrm{k}}-3-(-1)^{\mathrm{n}+\mathrm{k}}}{6} \\
& =\frac{2^{\mathrm{n}+2-\mathrm{k}}-3-(-1)^{\mathrm{n}+\mathrm{k}+1}}{6} .
\end{aligned}
$$

De modo análogo obtemos

$$
\begin{aligned}
\mathrm{B}_{\mathrm{n}+1, \mathrm{k}} & =\mathrm{A}_{\mathrm{n}, \mathrm{k}}+\mathrm{C}_{\mathrm{n}, \mathrm{k}} \\
& =\frac{2^{\mathrm{n}+1-\mathrm{k}}-3+(-1)^{\mathrm{n}+\mathrm{k}}}{6}+\frac{2^{\mathrm{n}+1-\mathrm{k}}+3+(-1)^{\mathrm{n}+\mathrm{k}}}{6} \\
& =\frac{2 \cdot 2^{\mathrm{n}+1-\mathrm{k}}+2 \cdot(-1)^{\mathrm{n}+\mathrm{k}}}{6} \\
& =\frac{2^{\mathrm{n}+1-\mathrm{k}}+(-1)^{\mathrm{n}+\mathrm{k}}}{3} .
\end{aligned}
$$


E também

$$
\begin{aligned}
\mathrm{C}_{\mathrm{n}+1, \mathrm{k}} & =\mathrm{B}_{\mathrm{n}, \mathrm{k}}+\mathrm{C}_{\mathrm{n}, \mathrm{k}} \\
& =\frac{2^{\mathrm{n}-\mathrm{k}}+(-1)^{\mathrm{n}+\mathrm{k}+1}}{3}+\frac{2^{\mathrm{n}+1-\mathrm{k}}+3+(-1)^{\mathrm{n}+\mathrm{k}}}{6} \\
& =\frac{2 \cdot 2^{\mathrm{n}-\mathrm{k}}+2 \cdot(-1)^{\mathrm{n}+\mathrm{k}+1}}{6}+\frac{2^{\mathrm{n}+1-\mathrm{k}}+3+(-1)^{\mathrm{n}+\mathrm{k}}}{6} \\
& =\frac{2^{\mathrm{n}+2-\mathrm{k}}+3+(-1)^{\mathrm{n}+\mathrm{k}+1}}{6} .
\end{aligned}
$$

Para concluir, vamos verificar que $\mathrm{A}_{\mathrm{n}, \mathrm{k}}+\mathrm{B}_{\mathrm{n}, \mathrm{k}}+\mathrm{C}_{\mathrm{n}, \mathrm{k}}=2^{\mathrm{n}-\mathrm{k}}$, pois essa soma corresponde ao total de vezes que o disco $\mathrm{k}$ passou pelos pinos $\mathrm{A}, \mathrm{B}$ e C, respectivamente, logo, deverá valer $2^{\mathrm{n}-\mathrm{k}}$.

$$
\begin{aligned}
\mathrm{A}_{\mathrm{n}, \mathrm{k}}+\mathrm{B}_{\mathrm{n}, \mathrm{k}}+\mathrm{C}_{\mathrm{n}, \mathrm{k}} & =\frac{2^{\mathrm{n}+1-\mathrm{k}}-3+2^{\mathrm{n}+1-\mathrm{k}}+2^{\mathrm{n}+1-\mathrm{k}}+3+2(-1)^{\mathrm{n}+\mathrm{k}}+2(-1)^{\mathrm{n}+\mathrm{k}+1}}{6} \\
& =\frac{3 \cdot 2^{\mathrm{n}+1-\mathrm{k}}+2(-1)^{\mathrm{n}+\mathrm{k}}+2(-1)^{\mathrm{n}+\mathrm{k}+1}}{6} \\
& =\frac{3 \cdot 2^{\mathrm{n}+1-\mathrm{k}}}{6} \\
& =\frac{2^{\mathrm{n}+1-\mathrm{k}}}{2} \\
& =2^{\mathrm{n}-\mathrm{k}} .
\end{aligned}
$$

Exemplo 1. Considerando o jogo das Torres de Hanói com 10 discos, quantas vezes o disco 1 foi movido para o pino $\mathrm{C}$ ?

Solução. Aplicando a fórmula (10) obtemos:

$$
\begin{aligned}
\mathrm{C}_{10,1} & =\frac{2^{10+1-1}+3+(-1)^{10+1}}{6} \\
& =\frac{1024+3-1}{6} \\
& =\frac{1026}{6} \\
& =171 .
\end{aligned}
$$

Logo, o disco 1 foi movido para o pino C 171 vezes.

Exemplo 2. Calcule quantas vezes o disco 4 foi movido para o pino B com um jogo de 6 discos.

Solução. Aplicando a fórmula (11) obtemos:

$$
\begin{aligned}
\mathrm{B}_{6,4} & =\frac{2^{6-4}+(-1)^{6+4+1}}{3} \\
& =\frac{4-1}{3} \\
& =1 .
\end{aligned}
$$


Exemplo 3. Calcule quantas vezes o disco 4 foi movido para o pino A com um jogo de 6 discos.

Solução. Aplicando a fórmula (12) obtemos:

$$
\begin{aligned}
A_{6,4} & =\frac{2^{6+1-4}-3+(-1)^{6+4}}{6} \\
& =\frac{8-3+1}{6} \\
& =1 .
\end{aligned}
$$

\section{Torre com 4 pinos - Número Mínimo de Movimentos}

Suponha que temos os $\mathrm{n}$ discos empilhados no pino A em forma de cone e desejamos transferilos para o pino D. Há várias formas de fazer essa transferência, mas estamos à procura da que minimiza a quantidade de movimentos. Denominaremos a quantidade mínima de movimentos necessária para mover os $\mathrm{n}$ discos de $\mathrm{b}_{\mathrm{n}}$.

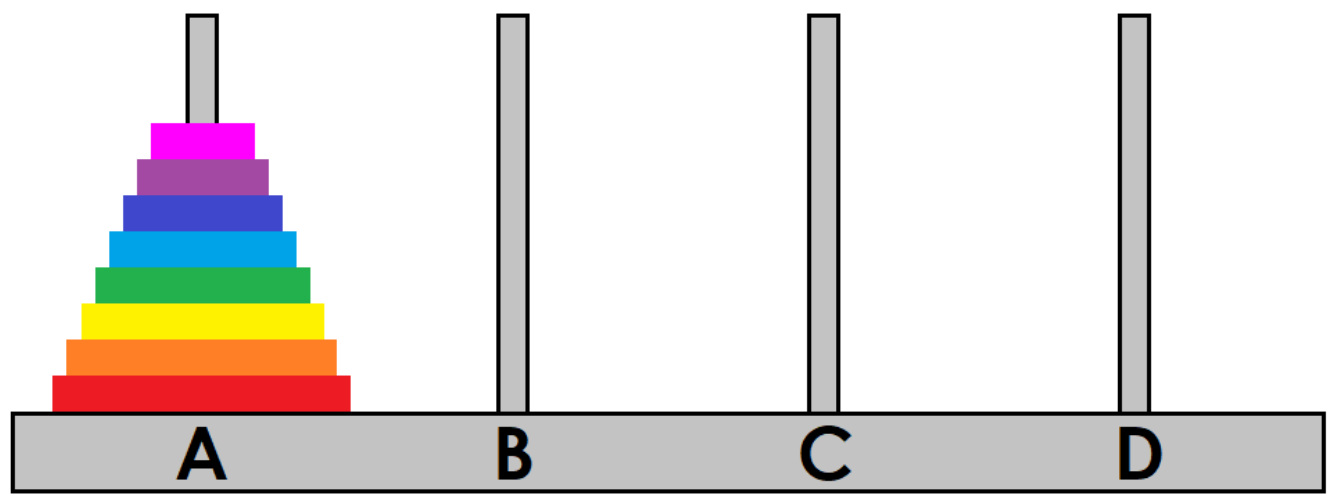

Figura 9: Torre de Hanói com 4 pinos.

De modo semelhante ao que foi feito no caso dos 3 pinos, observe que o último disco, ou seja, o maior de todos, será movido após retirarmos os $n-1$ discos que estão em cima dele e o distribuirmos nos pinos $\mathrm{B}$ e C. Ocorre que existem várias maneiras de tais discos estarem alocados nesses pinos B ou C, desse modo o caso da Torre com 4 pinos é distinto da Torre com 3 pinos, uma vez que nessa só há um modo dos pinos estarem distribuídos.

Vamos supor um método de solução e verificar se é o melhor. Para essse método, $\mathrm{x}_{\mathrm{n}}$ será a quantidade de movimentos para mover $\mathrm{n}$ discos de um pino para outro usando os 4 pinos. Imagine que coloquemos todos os $\mathrm{n}-1$ menores empilhados no pino $\mathrm{B}$; para isso usaremos $\mathrm{x}_{\mathrm{n}-1}$ movimentos. Então, transferimos o maior disco para o pino D usando 1 movimento, e para finalizar realocamos os $\mathrm{n}-1$ discos no pino $\mathrm{B}$ para o pino $\mathrm{D}$, usando mais $\mathrm{x}_{\mathrm{n}-1}$ movimentos e concluímos o jogo. Para isso, efetuamos $x_{n-1}+1+x_{n-1}$ movimentos no total, ou seja,

$$
\mathrm{x}_{\mathrm{n}}=2 \mathrm{x}_{\mathrm{n}-1}+1 \mathrm{e} \mathrm{x}_{1}=1
$$




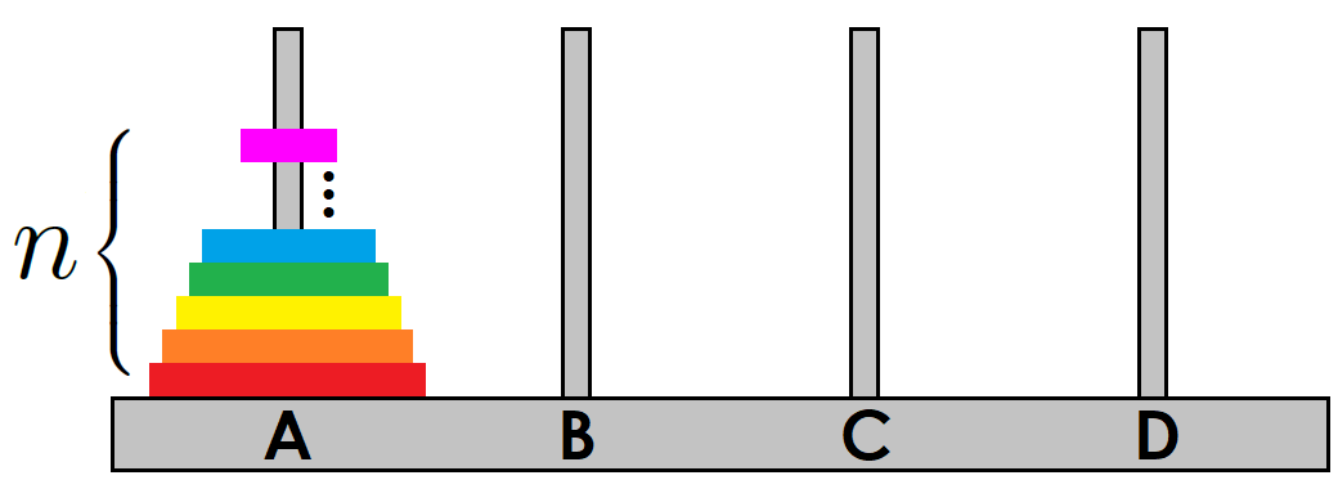

Figura 10: Torre de Hanói com 4 pinos.

pois com 1 disco, temos apenas 1 movimento que seria retirá-lo do pino A e colocá-lo do pino D, $\operatorname{logo}, x_{n}=2^{n}-1$. Será que essa é a menor quantidade de movimentos possíveis? Ou seja, $x_{n}=b_{n}$ ?

Vejamos outra situação possível: nesse caso chamaremos de $\mathrm{y}_{\mathrm{n}}$ a quantidade de movimentos para mover $\mathrm{n}$ discos. Imagine que transferimos os $\mathrm{n}-2$ discos menores para $\mathrm{B}$ usando $\mathrm{y}_{\mathrm{n}-2}$ movimentos, o segundo maior disco transferimos para $\mathrm{C}$ e o disco maior para D. Depois, transferimos o segundo disco maior para $\mathrm{D}$, e para terminar os $\mathrm{n}-2$ discos menores para D. Realizamos assim $\mathrm{y}_{\mathrm{n}-2}+1+$ $1+1+\mathrm{y}_{\mathrm{n}-2}$ movimentos, logo,

$$
\mathrm{y}_{\mathrm{n}}=2 \mathrm{y}_{\mathrm{n}-2}+3 \text { e } \mathrm{y}_{1}=1, \mathrm{y}_{2}=3
$$

uma recorrência diferente de (13). Vamos comparar os resultados para $\mathrm{n}=3$ discos. Como $\mathrm{y}_{1}=1$ e $\mathrm{y}_{2}=3$, então $\mathrm{y}_{3}=2 \mathrm{y}_{1}+3=5$. Na solução do parágrafo anterior tínhamos $\mathrm{x}_{3}=2^{3}-1=7$. Logo, a recorrência obtida dá-nos menos movimentos necessários que (13). Será que (14) é a recorrência procurada para 4 pinos? A resposta é não, vejamos por quê.

Para 6 discos, a solução de (14) será $\mathrm{y}_{6}=2 \mathrm{y}_{4}+3=2 \cdot\left(2 \mathrm{y}_{2}+3\right)+3=21$. Contudo, temos uma solução com menos movimentos: retirar os 3 menores discos de $\mathrm{A}$ e colocar em $\mathrm{B}$ usando $\mathrm{z}_{3}$ movimentos, mover os 3 maiores discos de A para $\mathrm{D}$ usando os pinos $\mathrm{A}, \mathrm{C}$ e $\mathrm{D}$; logo, usamos $\mathrm{a}_{3}=2^{3}-1=7$ movimentos. Por fim, movemos os 3 discos menores que estão em B para D usando z movimentos, totalizando $5+7+5=17$ movimentos. Assim, (14) está descartada também. 


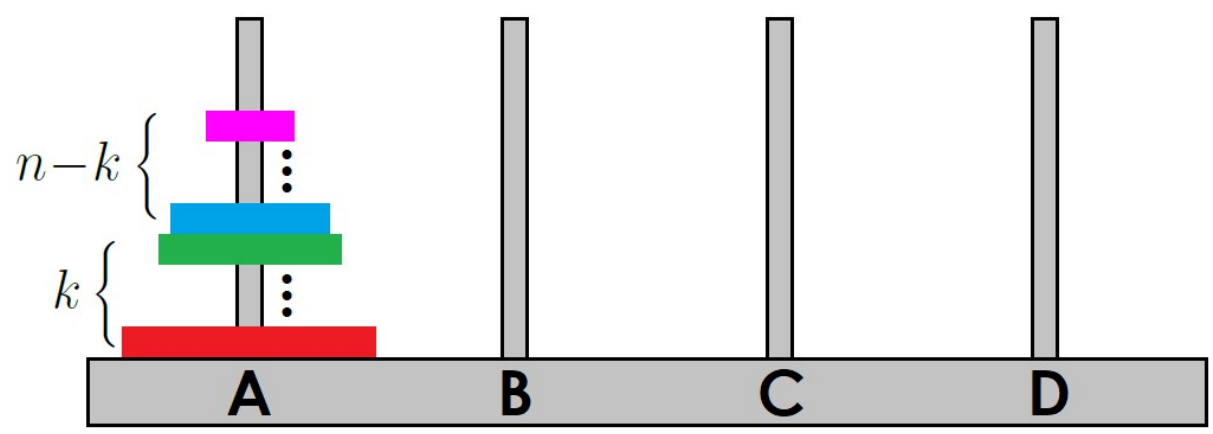

Figura 11: Torre de Hanói com 4 pinos.

Podemos concluir que o caso com 4 pinos é mais complexo do que o de 3 pinos, e a solução não é tão simples assim. Os resultados apresentados aqui estão em [1] com uma linguagem de programação. Observe que queremos encontrar a quantidade mínima de movimentos e observamos que dado um certo valor $\mathrm{k}, \mathrm{k}<\mathrm{n}$, usamos $\mathrm{b}_{\mathrm{n}-\mathrm{k}}$ movimentos para mover os $\mathrm{n}-\mathrm{k}$ discos menores para B; logo, a seguir movemos os discos restantes que são $\mathrm{k}$ para $\mathrm{D}$, para isso poderemos usar apenas os pinos $\mathrm{A}$, $\mathrm{C}$ e D, isto é, faremos $\mathrm{a}_{\mathrm{k}}$ movimentos, e para finalizar movemos os discos menores que estavam em $\mathrm{B}$ para $\mathrm{D}$, usando os 4 pinos, ou seja, $\mathrm{b}_{\mathrm{n}-\mathrm{k}}$ movimentos. Totalizando $\mathrm{b}_{\mathrm{n}}=2 \mathrm{~b}_{\mathrm{n}-\mathrm{k}}+\mathrm{a}_{\mathrm{k}}$ movimentos. Queremos encontrar o k que torna $b_{n}$ mínimo. Assim, queremos

$$
\mathrm{b}_{\mathrm{n}}=\min _{\mathrm{k} \leq \mathrm{n}}\left\{2 \mathrm{~b}_{\mathrm{n}-\mathrm{k}}+\mathrm{a}_{\mathrm{k}}\right\}=\min _{\mathrm{k} \leq \mathrm{n}}\left\{2 \mathrm{~b}_{\mathrm{n}-\mathrm{k}}+2^{\mathrm{k}}-1\right\} .
$$



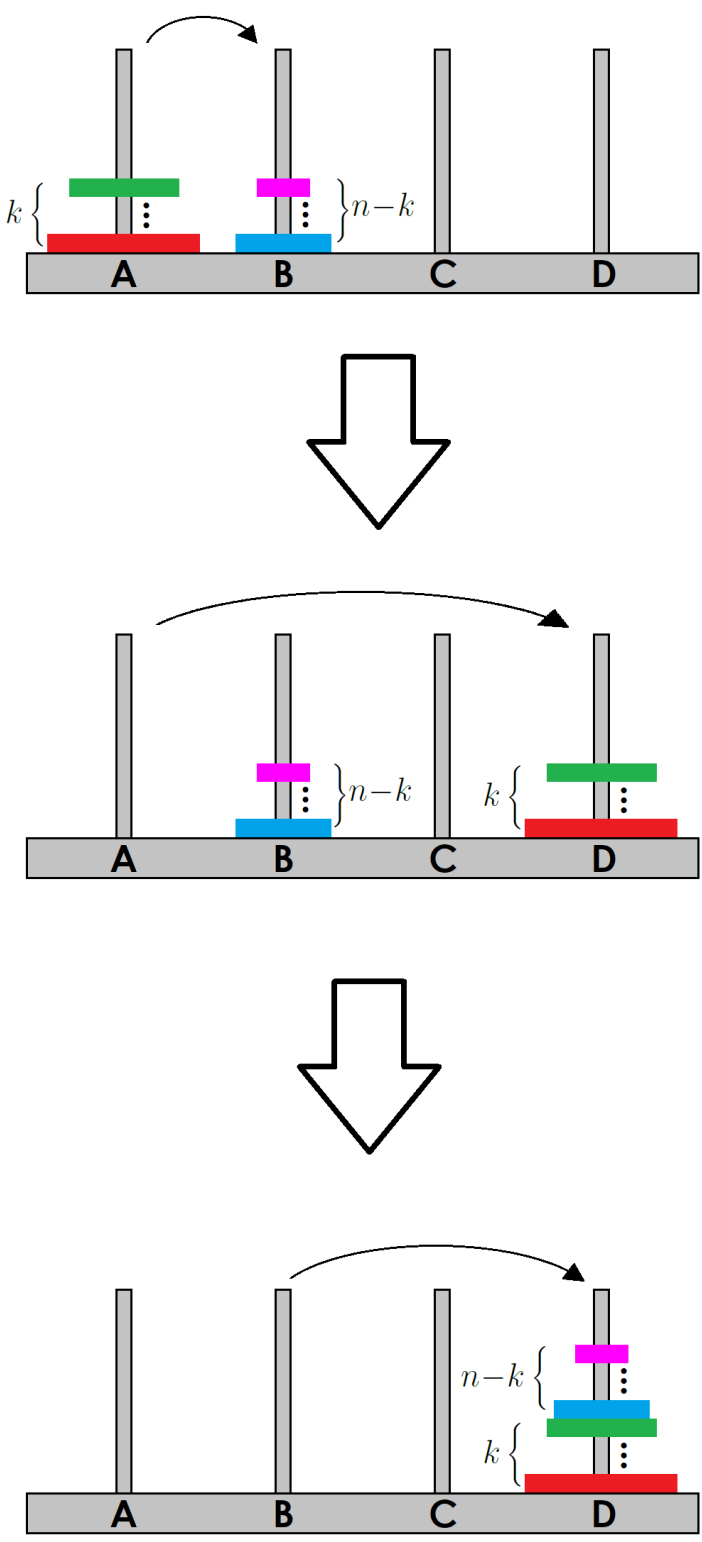

Figura 12: Torre de Hanói 4 pinos - Justificativa.

Analisando alguns casos: $\mathrm{n}=1,2,3,4,5,6,7,8,9,10,11,12,13,14,15$, observamos e levantaremos a hipótese de que os valores de $\mathrm{k}$ satisfazem a sequência

$$
\{1,1,2,2,2,3,3,3,3,4,4,4,4,4,5,5,5,5,5,5, \ldots\} \text {. }
$$

Se assim o for, dado n, precisamos saber sua posição na sequência, ou seja, o $k_{n}$. Sejam $\lfloor x\rfloor$ o maior inteiro menor ou igual a $\mathrm{x}$, e $\lceil\mathrm{x}\rceil$ o menor inteiro maior ou igual a $\mathrm{x}$. 
Proposição 4. Seja a sequência $\left\{\mathrm{k}_{\mathrm{n}}\right\}=\{1,1,2,2,2,3,3,3,3,4,4,4,4,4,5,5,5,5,5,5, .$.$\} , então,$

$$
\mathrm{k}_{\mathrm{n}}=\left\lfloor\frac{\sqrt{8 \mathrm{n}+1}-1}{2}\right\rfloor
$$

Demonstração. Essa sequência é composta por blocos de repetição dos números naturais. O último número de cada bloco de repetição é igual à soma das quantidades de elementos de cada bloco anterior a ele, por exemplo,

$$
\mathrm{k}_{2}=1,2=2 \mathrm{k}_{5}=2,5=3+2, \mathrm{k}_{9}=3,9=4+3+2 .
$$

Logo, a soma da quantidade de números de cada bloco resulta na última posição do bloco. Dado $\mathrm{n}$, para sabermos o $\mathrm{k}_{\mathrm{n}}$ correspondente a ele, resolvemos

$$
\frac{\mathrm{k}_{\mathrm{n}}\left(\mathrm{k}_{\mathrm{n}}+1+2\right)}{2}=\mathrm{n}
$$

Obviamente essa igualdade nem sempre é satisfeita; devemos resolver a equação do segundo grau

$$
\mathrm{k}_{\mathrm{n}}^{2}+3 \mathrm{k}_{\mathrm{n}}-2 \mathrm{n}=0 \Rightarrow \mathrm{k}_{\mathrm{n}}=\frac{-3+\sqrt{9+8 \mathrm{n}}}{2} .
$$

Se o valor de $\mathrm{k}_{\mathrm{n}}$ for inteiro, então o número mínimo de movimentos será $\mathrm{b}_{\mathrm{n}}=2 \mathrm{~b}_{\mathrm{n}-\mathrm{k}_{\mathrm{n}}}+\mathrm{a}_{\mathrm{k}_{\mathrm{n}}}$. Caso não seja inteiro, então,

$$
\mathrm{k}_{\mathrm{n}}=\left\lceil\frac{\sqrt{8 \mathrm{n}+9}-3}{2}\right\rceil=\left\lfloor\frac{\sqrt{8 \mathrm{n}+1}-1}{2}\right\rfloor,
$$

esse último valor é mais simplificado e $\frac{\sqrt{8 \mathrm{n}+1}-1}{2}-\frac{\sqrt{8 \mathrm{n}+9}-3}{2}<1$ para todo $\mathrm{n}$ natural.

Para provar o teorema principal dessa seção, precisamos do resultado a seguir.

Proposição 5. A sequência $\left\{\mathrm{b}_{\mathrm{n}}\right\}$ satisfaz a recorrência

$$
\mathrm{b}_{\mathrm{n}+1}=\mathrm{b}_{\mathrm{n}}+2^{\mathrm{k}_{\mathrm{n}}}
$$

Demonstração. Se temos $\mathrm{n}+1$ discos em $\mathrm{A}$, devemos retirar os $\mathrm{n}$ discos menores usando a menor quantidade de movimentos e alocá-los em B e C.

Se $b_{n}=2 b_{n-k_{n}}+a_{k_{n}}$, a solução para $n$ discos é transferir os $n-k_{n}$ discos menores para B usando os 4 pinos, depois os $\mathrm{k}_{\mathrm{n}}$ restantes para $\mathrm{C}$ (em vez de $\mathrm{D}$, caso convencional) usando os 3 pinos, e não faremos a última etapa, que é transferir os $n-k_{n}$ discos para $C$; assim usaremos $b_{n-k_{n}}+a_{k_{n}}$ movimentos.

Agora, transferiremos o disco $\mathrm{n}+1$ para $\mathrm{D}$, os $\mathrm{k}_{\mathrm{n}}$ que estão em $\mathrm{C}$ para $\mathrm{D}$, e, para terminar, os $\mathrm{n}-\mathrm{k}_{\mathrm{n}}$ que estão em B para D, usando $1+\mathrm{a}_{\mathrm{k}_{\mathrm{n}}}+\mathrm{b}_{\mathrm{n}-\mathrm{k}_{\mathrm{n}}}$. Logo, usamos $2 \mathrm{~b}_{\mathrm{n}-\mathrm{k}_{\mathrm{n}}}+2 \mathrm{a}_{\mathrm{k}_{\mathrm{n}}}+1$ movimentos. Assim, $b_{n+1}=2 b_{n-k_{n}}+2 a_{k_{n}}+1=2 b_{n-k_{n}}+a_{k_{n}}+a_{k_{n}}+1=b_{n}+a_{k_{n}}+1=b_{n}+2^{k_{n}}$.

O seguinte resultado dá-nos uma fórmula fechada para o número mínimo de movimentos da torre com $n$ discos e 4 pinos. 
Teorema 3. Seja n o número de discos de um Torre de Hanói com 4 pinos. Se

$$
\mathrm{k}_{\mathrm{n}}=\left\lfloor\frac{\sqrt{8 \mathrm{n}+1}-1}{2}\right\rfloor \text {, }
$$

então,

$$
\mathrm{b}_{\mathrm{n}}=2^{\mathrm{k}_{\mathrm{n}}}\left(\mathrm{n}-1-\frac{\mathrm{k}_{\mathrm{n}}\left(\mathrm{k}_{\mathrm{n}}-1\right)}{2}\right)+1
$$

Demonstração. Suponha que o teorema é válido para n,, mostraremos por indução que ele é verdadeiro para $n+1$. De acordo com a proposição anterior $b_{n+1}=b_{n}+2^{k_{n}}$. Logo, por hipótese de indução, $b_{n+1}=2^{k_{n}}\left(n-1-\frac{k_{n}\left(k_{n}-1\right)}{2}\right)+1+2^{k_{n}}$. Há 2 possibilidades $k_{n+1}=k_{n}$ ou $k_{n+1}=k_{n}+1$. Se $\mathrm{k}_{\mathrm{n}+1}=\mathrm{k}_{\mathrm{n}}$, então,

$$
\mathrm{b}_{\mathrm{n}+1}=2^{\mathrm{k}_{\mathrm{n}}}\left(\mathrm{n}-1-\frac{\mathrm{k}_{\mathrm{n}}\left(\mathrm{k}_{\mathrm{n}}-1\right)}{2}\right)+1+2^{\mathrm{k}_{\mathrm{n}}}=2^{\mathrm{k}_{\mathrm{n}+1}}\left((\mathrm{n}+1)-1-\frac{\mathrm{k}_{\mathrm{n}+1}\left(\mathrm{k}_{\mathrm{n}+1}-1\right)}{2}\right)+1 .
$$

logo, o teorema é válido. Caso $\mathrm{k}_{\mathrm{n}+1}=\mathrm{k}_{\mathrm{n}}+1$, queremos mostrar que

$$
\mathrm{b}_{\mathrm{n}+1}=2^{\mathrm{k}_{\mathrm{n}}+1}\left((\mathrm{n}+1)-1-\frac{\left(\mathrm{k}_{\mathrm{n}}+1\right)\left(\mathrm{k}_{\mathrm{n}}+1-1\right)}{2}\right)+1
$$

Simplificando,

$$
\mathrm{b}_{\mathrm{n}+1}=2^{\mathrm{k}_{\mathrm{n}}+1}\left(\mathrm{n}-\frac{\left(\mathrm{k}_{\mathrm{n}}+1\right)\left(\mathrm{k}_{\mathrm{n}}\right)}{2}\right)+1 .
$$

Pela proposição anterior

$$
\mathrm{b}_{\mathrm{n}+1}=2^{\mathrm{k}_{\mathrm{n}}}\left(\mathrm{n}-1-\frac{\mathrm{k}_{\mathrm{n}}\left(\mathrm{k}_{\mathrm{n}}-1\right)}{2}\right)+1+2^{\mathrm{k}_{\mathrm{n}}}=2^{\mathrm{k}_{\mathrm{n}}}\left(\mathrm{n}-\frac{\mathrm{k}_{\mathrm{n}}\left(\mathrm{k}_{\mathrm{n}}-1\right)}{2}\right)+1,
$$

que será igual a (23) se, e somente se,

$$
\begin{aligned}
& 2 \mathrm{n}-\left(\mathrm{k}_{\mathrm{n}}+1\right)\left(\mathrm{k}_{\mathrm{n}}\right)=\mathrm{n}-\frac{\mathrm{k}_{\mathrm{n}}\left(\mathrm{k}_{\mathrm{n}}-1\right)}{2} \\
\Rightarrow & 4 \mathrm{n}-2\left(\mathrm{k}_{\mathrm{n}}+1\right)\left(\mathrm{k}_{\mathrm{n}}\right)=2 \mathrm{n}-\mathrm{k}_{\mathrm{n}}\left(\mathrm{k}_{\mathrm{n}}-1\right) \\
\Rightarrow & 2 \mathrm{n}-2\left(\mathrm{k}_{\mathrm{n}}+1\right)\left(\mathrm{k}_{\mathrm{n}}\right)+\mathrm{k}_{\mathrm{n}}\left(\mathrm{k}_{\mathrm{n}}-1\right)=0 \\
\Rightarrow & 2\left(\mathrm{k}_{\mathrm{n}}\right)\left(\mathrm{k}_{\mathrm{n}}\right)-\mathrm{k}_{\mathrm{n}}\left(\mathrm{k}_{\mathrm{n}}-1\right)-2 \mathrm{n}= \\
\Rightarrow & \mathrm{k}_{\mathrm{n}}\left(2 \mathrm{k}_{\mathrm{n}}-\mathrm{k}_{\mathrm{n}}+1\right)-2 \mathrm{n}=0 \\
\Rightarrow & \mathrm{k}_{\mathrm{n}}\left(\mathrm{k}_{\mathrm{n}}+1\right)-2 \mathrm{n}=0 \\
\Rightarrow & \mathrm{k}_{\mathrm{n}}=\frac{-1+\sqrt{8 \mathrm{n}+1}}{2} .
\end{aligned}
$$


que comprova a veracidade do resultado.

Exemplo 4. Quantos movimentos são minimamente necessários para resolver o quebra-cabeça Torre de Hanói com 4 pinos quando temos 40 discos?

Solução. Nesse caso, temos

$$
\mathrm{k}_{40}=\left\lfloor\frac{\sqrt{8.40+1}-1}{2}\right\rfloor=8 \text {, }
$$

então

$$
\begin{aligned}
& b_{40}=2^{k_{40}}\left(40-1-\frac{\mathrm{k}_{40}\left(\mathrm{k}_{40}-1\right)}{2}\right)+1 \\
& \mathrm{~b}_{40}=2^{8}\left(39-\frac{8(8-1)}{2}\right)+1 \\
& b_{40}=2^{8} \cdot 11+1 \\
& b_{40}=2817 .
\end{aligned}
$$

\section{Referências}

[1] Chu, I-P.; Johnsonbaugh, R. The Four-Peg Tower of Hanoi Puzzle. SIGCSE. Vol. 23. N 3 Sept. 1991.

[2] Dudeney, H. E. The Canterbury Puzzles. New York: E. P. Dutton and co, 1908.

[3] Ferreira, D. B.; Oliveira, E. P. "A Matemática da Ordem das Movimentações das Peças da Torre de Hanói". Gazeta de Matemática, v. 1, N 190, pp. 6-10, 2020.

[4] Hinz, A. M.; Klavža, S.; Milutinović, U.; Petr, C. The Tower of Hanoi - Myths and Maths. Birkhäuser Basel, Springer, 2013.

[5] Lima, E. L.; Carvalho, P. C. P.; Wagner E.; Morgado, A. C. A Matemática do Ensino Médio. SBM, V. 1, 2012.

[6] Lucas, É. Récréations Mathématiques. Paris: Albert Blanchard, 1893.

[7] Morgado, A. C.; Carvalho, P. C. P. Matemática Discreta - Coleção Profmat. Rio de Janeiro: SBM, 2015.

[8] Oliveira, E. P. As Diversas Maneiras de Explorar a Matemática Através do Jogo Torres de Hanói. - Dissertação de Mestrado do Profmat, 2018.

[9] Pereira, A.; Rodrigues, R. "O problema das torres de Hanoi: a lenda, algoritmos e generalizações". Gazeta de Matemática, V. 1, N 144, pp. 10-11, 2003. 
Débora Borges Ferreira Universidade Federal do Rio Grande do Norte <debora@ccet.ufrn.br>

Edvan Pontes de Oliveira Escola Estadual em Tempo Integral Francisco de Assis Bittencourt $<$ edvan.pontes@hotmail.com>

Francisco Quaranta Neto Instituto Federal de Educação, Ciência e Tecnologia do Rio Grande do Norte $<$ francisco.quaranta@ifrn.edu.br>

Recebido: $27 / 04 / 2020$

Publicado: 07/12/2020 